\title{
Profili di sensibilità agli agenti antifungini di lieviti e miceti lievito-simili isolati negli ospedali torinesi
}

\author{
Vivian Tullio', Janira Roana', Narcisa Mandras', Giuliana Banche', Valeria Allizond', \\ Ornella Cervetti ${ }^{2}$, Michele Panzone ${ }^{2}$, Cristina Crocillà ${ }^{3}$, Ester Gaido ${ }^{4}$, Barbara Fianchino ${ }^{5}$, \\ Maria Agnese Latino', Anna Maria Cuffini', Nicola Carlone' \\ ' Dip. Sanità Pubblica e Microbiologia, Università degli Studi di Torino \\ ${ }^{2}$ Dip. Discipline Medico-Chirurgiche, Clinica Dermatologica II, Ospedale Dermatologico San Lazzaro, \\ Università degli Studi di Torino \\ ${ }^{3}$ Lab. Analisi Chimico-cliniche e Microbiologia, A. S. O. Ordine Mauriziano di Torino \\ ${ }^{4}$ Lab. Microbiologia, A. S. O. San Giovanni Battista (Molinette), Torino \\ ${ }^{5}$ Lab. Analisi Microbiologiche, Comprensorio Ospedaliero Amedeo di Savoia, Torino \\ ${ }^{6}$ Lab. Analisi Microbiologiche, A. S. O. O.I.R.M. - Sant'Anna, Torino
}

Key words: Yeasts, yeast-like fungi, antifungal agents, drug resistance.

Susceptibility profiles to current antifungal agents of clinical isolates of yeasts and yeast-like fungi from Turin hospitals.

\section{SUMMARY}

There is an increased incidence of the morbidity and mortality associated with fungal infections caused by resistant fungi in various groups of patients; therefore the monitoring of their susceptibility to antifungal agents to be necessary for optimizing clinical therapeutic treatment. The susceptibility profiles of recent clinical yeasts and yeast-like fungi isolated from Turin's Hospitals to current antifungal drugs are here reported. Candida albicans was the most frequently isolated species ( $40 \%)$, followed by C. glabrata (22\%). A high rate of susceptibility to all antifungal agents tested was observed and low evidence of resistance was found.

\section{INTRODUZIONE}

L'aumento del numero delle infezioni da funghi in ambiente ospedaliero nell'ultimo decennio costituisce un problema preoccupante, in quanto le infezioni nosocomiali hanno, nell' $8 \%$ dei casi, un'origine fungina, in particolare da Candida spp. $(2,12)$.

L'uso sempre più frequente di terapie immunosoppressive e di tecniche di rianimazione sempre più invasive, la comparsa di nuove patologie che comportano la compromissione delle difese immunitarie, l'aumento del numero dei trapianti, ecc. determinano lo sviluppo delle infezioni fungine che colpiscono, in particolare, i malati chirurgici ed i pazienti cancerosi sottoposti a chemioterapia, nei quali le candidosi invasive si accompagnano ad un elevato tasso di mortalità $(4,12)$.

A causa dell'emergere dell'AIDS, la candidosi oro-faringea interessa, ancora oggi, la quasi totalità dei malati con $\operatorname{HIV}(18,26)$.

Anche per quanto riguarda un altro lievito, il Cryptococcus neoformans, il numero dei casi diagnosticati sta progressivamente aumentando dal 1985 (dal 20 al 50\% per anno), a seguito del progressivo sviluppo dell'AIDS (27).

Infine, nei soggetti che non presentano anomalie immunitarie o particolari fattori di rischio, le infezioni da lieviti provocano focolai cutaneo-mucosi che, per quanto a carattere benigno, costituiscono una reale fonte di disagio.

Circa il $40 \%$ delle dermatomicosi è causato da Candida albicans e, secondo la letteratura, si può stimare in circa il 5\% il numero delle infezioni vulvo-vaginali causate da lieviti: il carattere spesso recidivante può evolvere in patologie così gravi da provocare invalidità al lavoro e notevole crescita dei costi pubblici e privati.

Di conseguenza, l'aumentata frequenza delle infezioni da lieviti e la gravità della loro evoluzione clinica, impone che qualsiasi fungo isolato da campioni clinici debba essere considerato come un potenziale opportunista, per il quale occorre eseguire un'identificazione accurata e sarebbe auspicabile determinare la sua sensibilità ai farmaci antifungini, soprattutto per quei miceti isolati da distretti sterili.

Scopo dello studio è stato pertanto quello di identificare i lieviti isolati da diversi campioni patologici, provenienti da distretti sterili e non sterili sia di soggetti immunocompetenti, sia di soggetti immunodepressi afferenti ai vari Ospedali della Città di Torino e di valutare la loro sensibilità ai più comuni farmaci antifungini.

\section{MATERIALI E METODI}

Identificazione dei lieviti. I vari ceppi fungini provenienti da cinque centri ospedalieri torinesi 
da marzo 2002 a febbraio 2003 (Ospedale Amedeo di Savoia, Mauriziano, O.I.R.M. Sant'Anna, Dermatologico San Lazzaro e San Giovanni Battista - Molinette) sono stati identificati mediante prove biochimiche di assimilazione degli zuccheri e substrati vari, utilizzando le gallerie del sistema API ID 32C (bioMérieux, Roma, Italia).

Test di sensibilità agli antifungini. Il test è stato effettuato con la tecnica di diffusione in agar con dischetti utilizzando il sistema commerciale ATB fungal system (Biogenetics Diagnostics, Ponte San Nicolò, PD, Italia) ed il metodo messo a punto da Meis et al. (17).

\section{ATB fungal system.}

L'inoculo fungino è stato preparato in acqua distillata alla densità di $0.5 \mathrm{McF}$ arland, corrispondente a $1-5 \times 10^{6}$ lieviti $/ \mathrm{ml}$ e ulteriormente diluito 1:10 per ottenere l'inoculo finale di $10^{5} \mathrm{lieviti} / \mathrm{ml}$. Il terreno utilizzato è stato il Fungal ATB Medium (Biogenetics) inoculato con $5 \mathrm{ml}$ della sospensione di lieviti.

Dopo aver applicato i mycodisk sulla superficie dell'agar, le piastre sono state lasciate a temperatura ambiente per 30' affinché i composti antifungini diffondessero nel terreno e poi incubate a $37^{\circ} \mathrm{C}$ per $24-48$ ore.

Come controllo di qualità è stata utilizzata Candida albicans ATCC 90028.

\section{Metodo di Meis et al.}

L'inoculo fungino è stato preparato in soluzione salina allo $0.9 \%$, fino ad ottenere una sospensione pari a 0.5 McFarland.

Il terreno utilizzato è stato il Müeller Hinton agar (MHA) addizionato di glucosio al $2 \%$ e di 5 $\mathrm{mg} / \mathrm{ml}$ di blu di metilene, che favoriscono la crescita e migliorano la definizione del margine degli aloni di inibizione. Le piastre sono state incubate in aerofilia a $35^{\circ} \mathrm{C}$ per $24-48$ ore.

Antimicotici. Sono stati saggiati i seguenti antifungini del commercio: anfotericina B $(20 \mu \mathrm{g}$ e $100 \mu \mathrm{g})$ nistatina (100 U.I.), 5-fluorocitosina $(1 \mu \mathrm{g}$ e $10 \mathrm{mg})$, griseofulvina $(25 \mu \mathrm{g})$, ciclopiroxolamina $(50 \mathrm{mg})$, miconazolo, econazolo, clotrimazolo, isoconazolo, ketoconazolo $(100 \mu \mathrm{g})$ (Biogenetics) e fluconazolo $(100 \mu \mathrm{g})$ (Liofilchem, Roseto, TE, Italia). Ad eccezione del fluconazolo, tutti gli antimicotici erano inseriti nel kit del sistema micodisk (ATB fungal system).

Per quanto riguarda il fluconazolo, il test di sensibilità è stato eseguito in parallelo confrontando le due metodiche ed utilizzando quindi come terreno il Fungal ATB Medium (FLU ${ }_{100 a f m}$ ) ed il MHA contenente il $2 \%$ di glucosio e $5 \mu \mathrm{g} / \mathrm{ml}$ di blu di metilene (FLU $\left.{ }_{100 \mathrm{mha}}\right)$.

Interpretazione. Ai fini interpretativi, è stata uti- lizzata la tabella 1.

Per il fluconazolo è stato usato sia il range di sensibilità per gli azoli $(\mathrm{S} \geq 20 \mathrm{~mm}, \mathrm{R} \leq 19 \mathrm{~mm})$ fornito dalla ditta produttrice (Liofilchem, ) che quello del metodo di Meis et al. $(\mathrm{S} \geq 19 \mathrm{~mm}$, Susceptible Dose Dependent $=13-18 \mathrm{~mm}, \mathrm{R} \leq 12$ $\mathrm{mm}$ ), basato sui break-point delle MIC raccomandati dall'US-NCCLS $(19,20)$.

Per SDD si intende sensibilità dose dipendente e corrisponde per i funghi alla categoria sensibilità intermedia usata per i batteri; tuttavia, sottolinea la necessità di aumentare la dose di farmaco per ottenere sensibilità.

\section{RISULTATI}

In questo lavoro sono stati isolati 114 ceppi di lieviti e lievito-simili provenienti da 5 ospedali presenti nella città di Torino: Ospedale Amedeo di Savoia (26 ceppi), Ospedale Mauriziano (25 ceppi), Ospedale O.I.R.M. - Sant'Anna (23 ceppi), Ospedale Dermatologico San Lazzaro (21 ceppi) ed Ospedale San Giovanni Battista Molinette (19 ceppi). Sono state trovate 13 specie differenti di Candida spp. (corrispondenti all' $85 \%$ dei ceppi isolati) tra le quali quella più rappresentativa è stata $C$. albicans, presente tra tutti i ceppi isolati al $40 \%$ e tra i ceppi di Candida spp. al 45\% (tabella 2). In percentuale minore si sono ritrovati: C. glabrata (22\%); C. parapsilosis (7\%); C. krusei (4\%); C. famata, C. guilliermondii e C. tropicalis (2\%); C. humicola, C.intermedia, C. kefyr, C. lypolitica, C. pulcherrima e $C$. valida (1\%). Altri ceppi isolati sono stati: C. neoformans (2\%), Saccharomyces cerevisiae (9\%), Trichosporon cutaneum (4\%), Rhodotorula spp. e Pichia carsonii (1\%).

Questi risultati rientrano nell'andamento riscontrato nei diversi paesi europei ed extra-europei nei quali la maggior parte dei lieviti patogeni o potenzialmente patogeni ed i lievito-simili isolati in laboratorio appartiene a cinque generi principali (Candida, Cryptococcus, Rhodotorula, Saccharomyces, Trichosporon o, molto più raramente, altre forme sessuate di lieviti come Hansenula, ecc.), a cui occorre aggiungere Geotrichum spp. che, quantunque si tratti di un fungo filamentoso e non di un lievito, cresce sotto forma di colonie simili e viene isolato nel medesimo contesto. Globalmente ciò corrisponde a circa 40-50 specie differenti, di cui circa quindici sono tra le più frequenti.

Poiché nel sistema disponibile commercialmente sono presenti antifungini non più utilizzati, come la ciclopiroxolamina o utilizzati nei confronti di altri miceti, come la griseofulvina, i risultati ottenuti per questi farmaci non sono stati presentati (tabella 3).Tutti i miceti saggiati, presenti nei 
campioni provenienti dai vari ospedali, sono risultati sensibili all'anfotericina $\mathrm{B}\left(\mathrm{AMP}_{20}\right.$, AMP ${ }_{100}$ ) e alla nistatina (NYS) (tabella 3 ).

Le specie di Candida (comprese quelle non albicans) sono risultate per lo più tutte sensibili alla 5-fluorocitosina alle due diverse concentrazioni $\left(5 \mathrm{FC}_{1}\right.$ e $\left.5 \mathrm{FC}_{10}\right)$, con alcuni casi di sensibilità intermedia o di resistenza. Per quanto riguarda gli imidazoli (MIC, ECO, CLO, ISO, KET) tutti i ceppi in esame, ad eccezione di C. albicans, sono risultati sensibili o mediamente sensibili a questi antimicotici.

Tra i i ceppi di C. albicans si sono riscontrati, oltre al miconazolo, casi di resistenza o sensibilità intermedia verso uno o più derivati imidazolici, soprattutto nei confronti del miconazolo, clotrimazolo e ketoconazolo.

Gli altri miceti non Candida saggiati quali C. neoformans e $S$. cerevisiae sono risultati sensibili alla 5-fluorocitosina alle due diverse concentrazioni e a tutti gli imidazoli saggiati, ad eccezione di tre ceppi di Saccharomyces, che sono risultati resistenti al clotrimazolo. Un ceppo, inoltre, è risultato poco sensibile anche a miconazolo ed econazolo (tabella 3).

Per quanto riguarda T. cutaneum tre ceppi su quattro hanno manifestato resistenza alla 5-fluorocitosina, mentre uno ha mostrato sensibilità intermedia a questo antimicotico.

Tutti i Trichosporon sono risultati sensibili agli imidazoli, ad eccezione di un caso di sensibilità intermedia al miconazolo. In ultima analisi, i ceppi di P. carsonii e di Rhodotorula spp. si sono mostrati sensibili a tutti gli antimicotici saggiati.

Su 47 ceppi di miceti (11 C. albicans, 12 C. glabrata, 1 C. famata, 1 C. kefyr, 2 C. krusei, 1 C. guilliermondii, 1 C. humicola, 1 C. intermedia, 3 C. parapsilosis, 1 C. tropicalis, 1 C. lipolytica, 2 C. neoformans, 1 P. carsonii, $5 \mathrm{~S}$. cerevisiae, $4 T$. cutaneum) è stata valutata la sensibilità al fluconazolo, utilizzando come terreno base per l'antimicogramma il MHA, contenente $5 \mu \mathrm{g} / \mathrm{ml}$ di blu di metilene e glucosio al 2\% (FLU (100ma $)$, e il Fungal ATB Medium (FLU $100 a$ afm). Dal confronto tra le due metodiche è emerso che, per le specie di Candida spp., i due risultati non concordano nella sensibilità o resistenza verso questo farmaco (tabella 3): infatti, si sono riscontrati 21 ceppi dotati di resistenza per il FLU 100afm $_{\text {ma di sensibili- }}$

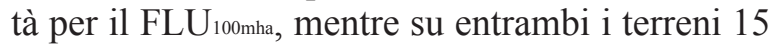
sono risultati resistenti. Per quanto riguarda le specie non candida, C. neoformans (2 ceppi), $P$. carsonii (1 ceppo) e un ceppo su cinque di $S$. cerevisiae, si sono rivelati sensibili al fluconazolo su entrambi i terreni (gli altri 4 ceppi di S. cerevisiae si sono rivelati resistenti per il FLU ${ }_{100 a f m}$ ma sensibili per il FLU100ma) (tabella 3). Dei ceppi di
T. cutaneum saggiati, invece, due sono risultati sensibili ed uno resistente su entrambi i terreni mentre uno si è rivelato sensibile al fluconazolo sul terreno MHA ma resistente sul terreno AFM (tabella 3).

\section{DISCUSSIONE E CONCLUSIONI}

Una delle principali sfide della terapia antifungina è lo sviluppo di tecniche standardizzate per studiare la sensibilità in vitro agli agenti antifungini che abbiano una rilevanza clinica e che possano determinare la vera significatività della resistenza clinica a questi farmaci.

$\mathrm{Ci}$ sono quattro motivi principali per cui attualmente questi test presentano un crescente interesse:

a) l'aumento dei soggetti potenzialmente a rischio di sviluppare un'infezione da funghi opportunisti che, oltre ai soggetti traumatizzati e diabetici, colpisce soggetti immunocompromessi;

b) il numero crescente di specie fungine considerate saprofite che, attualmente in pazienti immunocompromessi, sono isolate ripetutamente da infezioni sistemiche, ascessi cerebrali, ecc., e la cui sensibilità agli antifungini è sconosciuta (22);

c) l'incremento del numero di farmaci antifungini ad ampio spettro disponibili, mentre altri sono in via di sviluppo;

d) l'osservazione, sia clinica che di laboratorio, di resistenze secondarie di alcuni lieviti dopo terapie con anfotericina B o fluconazolo (3). Alcune specie come C. glabrata e C. krusei sono di solito poco sensibili o addirittura resistenti agli antifungini della serie degli azoli (ketoconazolo e fluconazolo). Inoltre, si assiste all'emergere di ceppi di $C$. lusitaniae e anche di C. albicans meno sensibili all'anfotericina B.

Attualmente, le infezioni da funghi patogeni non sono più riconducibili solamente a pazienti gravemente immunocompromessi a causa di tumori ematici o perché trapiantati, ma si sviluppano anche in pazienti debilitati che sono stati ricoverati in reparti di terapia intensiva o in reparti con un'alta frequenza di ricoveri $(2,24)$.

La maggior parte di queste infezioni è causata da C. albicans che, come risulta anche dal presente lavoro, è la specie isolata con maggior frequenza nei vari ospedali e quindi considerata più aggressiva $(9,16)$. Ceppi non albicans sono comunque responsabili del $35-65 \%$ delle candidosi riscontrate nei pazienti ospedalizzati.

I ceppi più comuni sono dati da C. parapsilosis, C. krusei, C. glabrata $(5,14)$, ma stanno emergendo anche C. lusitaniae e C. guilliermondii, lieviti caratterizzati da una diminuzione di sensibilità nei confronti dei più comuni antimicotici $(6$, $10,24)$. Secondo i più recenti dati della letteratu- 
ra (14), C. famata e C. kefyr sono lieviti ancora poco diffusi ed isolabili con percentuale inferiore all' $1 \%$, anche se nella nostra indagine sono stati rinvenuti, rispettivamente, nel $2 \%$ e $1 \%$ dei casi, indicando anche qui una tendenza all'aumento.

La sensibilità in vitro di un microrganismo infettante al farmaco antimicotico utilizzato per l'eradicazione della malattia, è uno dei molti fattori che influenzano la probabilità che tale terapia abbia successo. Dopo circa trent'anni di studio, la sensibilità in vitro ad un chemioantibiotico può essere in grado di predire la risposta alle infezioni batteriche con un'accuratezza riassumibile con la regola del 90-60: le infezioni dovute a ceppi sensibili rispondono alla terapia nel $90 \%$ dei casi, mentre le infezioni dovute a ceppi resistenti corrispondono al $60 \%$. Un valore simile si è mostrato predittivo anche per $\mathrm{i}$ test di sensibilità nei confronti dei lieviti e nella conseguente scelta degli antimicotici più idonei (23). La terapia corrente nei confronti dei lieviti prevede l'uso dei seguenti antimicotici: i polieni, che interagiscono con gli steroli presenti a livello della membrana fungina; la 5-fluorocitosina, che inibisce la sintesi delle macromolecole indispensabili per la sopravvivenza del micete; gli imidazoli e gli azoli che inibiscono la sintesi di ergosterolo, componente fondamentale della membrana fungina (11).

Tra questi farmaci i primi tre risultano essere i più efficaci, in quanto, tra tutti i miceti esaminati, soltanto 6 ceppi di Candida spp.(due di C. krusei, uno di C. guilliermondii, due di C. albicans e uno C. parapsilosis) e quattro ceppi di T. cutaneum hanno dimostrato di possedere sensibilità intermedia o resistenza. Questi risultati trovano conferma in letteratura nei lavori compiuti da Alvarado et al. (1) con C. albicans e C. parapsilosis e da Rogers (24) con ceppi di Candida spp. nei confronti dell'anfotericina $\mathrm{B}$.

Nel caso degli imidazoli, invece, si osserva l'insorgenza di un numero maggiore di resistenze in accordo con quanto visto in un recente lavoro di St. Georgiev (25), probabilmente dovute a cicli di terapia prolungati (più di 3 anni) (5). Tra i farmaci appartenenti a questa classe, quelli risultati "meno efficaci" sono stati il miconazolo (43 ceppi dotati di sensibilità intermedia o di resistenza tra cui 4 di C. glabrata, 33 di $C$. albicans, 1 di $C$. humicola, 1 di C. parapsilosis, 1 di C. tropicalis, 1 di C. valida, 1 di T. cutaneum e 1 di S. cerevisiae), il clotrimazolo (36 ceppi con sensibilità intermedia o resistenti tra cui 21 di C. albicans), il ketoconazolo (24 ceppi con sensibilità intermedia 0 resistenti tra cui 23 di C. albicans ed 1 di C. krusei). Per quanto riguarda il fluconazolo, saggiato soltanto su 47 ceppi, le sensibilità riscontrate sia su FLU ${ }_{100 m h a}$ che su FLU100afm sono riferibili solo a
9 ceppi fungini (2 di T. cutaneum, 2 di C. neoformans, 2 di C. parapsilosis, 1 di P. carsonii, 1 di $C$. albicans, 1 di $S$. cerevisiae), mentre la sensibilità su FLU ${ }_{100 m h a}$ si riferisce a 32 ceppi, in particolare 3 di C. parapsilosis, 3 di T. cutaneum, 1 di C. intermedia, 7 di C. glabrata, 9 di C. albicans, 1 di $C$. kefyr, 5 di S. cerevisiae, 2 di C. neoformans e 1 di $P$. carsonii. Le differenze nella sensibilità al fluconazolo riscontrate utilizzando due terreni diversi (MHA e AFM) sottolineano ancora una volta l'importanza di usare metodi sempre più standardizzati per valutare la sensibilità ai farmaci antifungini. Studi recenti di sorveglianza internazionale dimostrano che la maggior parte dei lieviti è ancora sensibile al fluconazolo $(7,10,15,17)$ e, di conseguenza, avvalorano il metodo di Meis et al. approvato, recentemente, dall'NCCLS (20). Tuttavia, per avere un quadro più completo, sarebbero necessari ulteriori studi, volti a valutare nel tempo l'andamento della resistenza a questi farmaci. Inoltre, anche se la resistenza tendesse a stabilizzarsi su quest'andamento si renderebbe comunque necessaria l'introduzione di nuovi farmaci antifungini, come ad esempio i nuovi triazoli (voriconazolo, posaconazolo, ranuconazolo) caratterizzati da una maggiore attività nei confronti dei miceti, da minore tossicità, da una maggiore accessibilità biologica e da una maggiore emivita $(8,13,21)$.

Tabella I. Range di sensibilità agli agenti antifungini

\begin{tabular}{|c|c|c|}
\hline Antimicotico & $\mathrm{mm}$ & S I R \\
\hline \multirow[t]{3}{*}{ 5-fluorocitosina $1 \mu \mathrm{g}$} & $\geq 20$ & $\mathrm{~S}$ \\
\hline & $19-11$ & I \\
\hline & $\leq 10$ & $\mathrm{R}$ \\
\hline \multirow[t]{2}{*}{ 5-fluorocitosina $10 \mu \mathrm{g}$} & $\geq 10$ & $\mathrm{~S}$ \\
\hline & $<10$ & $\mathrm{R}$ \\
\hline \multirow{2}{*}{$\begin{array}{l}\text { anfotericina B } 20 \mu \mathrm{g} \\
\text { anfotericina B } 100 \mu \mathrm{g}\end{array}$} & $>10$ & $\mathrm{~S}$ \\
\hline & $\leq 10$ & $\mathrm{R}$ \\
\hline \multirow[t]{2}{*}{ nistatina 100 U.I. } & $>10$ & $\mathrm{~S}$ \\
\hline & $\leq 10$ & $\mathrm{R}$ \\
\hline \multirow{4}{*}{$\begin{array}{c}\text { econazolo } 50 \mu \mathrm{g} \\
\text { clotrimazolo } 50 \mu \mathrm{g} \\
\text { miconazolo } 50 \mu \mathrm{g} \\
\text { ketoconazolo } 50 \mu \mathrm{g} \\
\text { isoconazolo } 50 \mu \mathrm{g}\end{array}$} & & \\
\hline & $\geq 20$ & $\mathrm{~S}$ \\
\hline & $19-11$ & I \\
\hline & $\leq 10$ & $\mathrm{R}$ \\
\hline \multirow{3}{*}{ ciclopiroxolamina $50 \mu \mathrm{g}$} & $\geq 20$ & $\mathrm{~S}$ \\
\hline & $19-11$ & I \\
\hline & $\leq 10$ & $\mathrm{R}$ \\
\hline \multirow[t]{2}{*}{ griseofulvina $25 \mu \mathrm{g}$} & $\geq 10$ & $\mathrm{~S}$ \\
\hline & $\leq 10$ & $\mathrm{R}$ \\
\hline
\end{tabular}


Tabella 2. Frequenza di isolamento dei lieviti e lievito-simili negli ospedali torinesi

\begin{tabular}{|c|c|c|c|c|c|c|}
\hline \multicolumn{7}{|c|}{ OSPEDALI TORINESI } \\
\hline & ADS & OM & OIRM & SL & MOL & \\
\hline MICETI & $n^{\circ}$ ceppi $=26^{*}$ & $n^{\circ}$ ceppi ${ }^{2}=25^{*}$ & $\mathrm{n}^{\circ}$ серpi $=23^{*}$ & $n^{\circ}$ ceppi $=2 I^{*}$ & $n^{\circ}$ серpi $=19 *$ & $\mathrm{n}^{\circ}$ ceppi tot $=114^{* *}$ \\
\hline C. albicans & $45 \%$ & $52 \%$ & $36 \%$ & $18 \%$ & $47 \%$ & $40 \%$ \\
\hline C. glabrata & $15 \%$ & $28 \%$ & $17 \%$ & 1 & $53 \%$ & $22 \%$ \\
\hline S. cerevisiae & $4 \%$ & $8 \%$ & $26 \%$ & $5 \%$ & 1 & $9 \%$ \\
\hline C. parapsilosis & $12 \%$ & 1 & $4 \%$ & $18 \%$ & 1 & $7 \%$ \\
\hline C. krusei & $8 \%$ & 1 & $13 \%$ & 1 & 1 & $4 \%$ \\
\hline T. cutaneum & 1 & 1 & 1 & $19 \%$ & 1 & $4 \%$ \\
\hline C. guilliermondii & $4 \%$ & 1 & 1 & $19 \%$ & 1 & $2 \%$ \\
\hline C. tropicalis & 1 & 1 & $4 \%$ & $5 \%$ & 1 & $2 \%$ \\
\hline C. famata & 1 & 1 & 1 & $10 \%$ & 1 & $2 \%$ \\
\hline Cr. neoformans & $8 \%$ & 1 & 1 & 1 & 1 & $2 \%$ \\
\hline C. lipolytica & 1 & 1 & 1 & $5 \%$ & 1 & $1 \%$ \\
\hline C. pulcherrima & $4 \%$ & 1 & 1 & 1 & 1 & $1 \%$ \\
\hline C. valida & 1 & $4 \%$ & 1 & 1 & 1 & $1 \%$ \\
\hline C. intermedia & 1 & 1 & 1 & $5 \%$ & 1 & $1 \%$ \\
\hline C. humicola & 1 & 1 & 1 & $5 \%$ & 1 & $1 \%$ \\
\hline P. carsonii & 1 & $4 \%$ & 1 & 1 & 1 & $1 \%$ \\
\hline Rhodotorula spp. & 1 & 1 & 1 & $5 \%$ & 1 & $1 \%$ \\
\hline C. kefyr & 1 & $4 \%$ & 1 & 1 & 1 & $1 \%$ \\
\hline
\end{tabular}

* \% calcolate sulla base del $n^{\circ}$ dei ceppi per singolo ospedale

** \% calcolate sulla base del $n^{\circ}$ totale dei ceppi isolati per singola specie

ADS = Amedeo di Savoia

$O M=$ Ordine Mauriziano

OIRM = OIRM-Sant'Anna

SL = Dermatologico San Lazzaro

MOL $=$ S. Giovanni Battista (Molinette)

Tabella 3. Proflii di sensibilità agli antimicotici

\begin{tabular}{|c|c|c|c|c|c|c|c|c|c|c|c|c|c|c|c|c|c|c|c|c|c|c|c|c|c|c|c|c|c|c|c|c|c|c|}
\hline \multirow[b]{2}{*}{ Ceppi isolati e saggiati } & \multicolumn{3}{|c|}{ AMP $_{20}$} & \multicolumn{2}{|c|}{$\mathrm{AMP}_{100}$} & \multicolumn{3}{|c|}{ NYS } & \multicolumn{3}{|c|}{$5 F C_{1}$} & \multicolumn{3}{|c|}{$5 \mathrm{FC}_{10}$} & \multicolumn{3}{|c|}{ MIC } & \multicolumn{3}{|c|}{ ECO } & \multicolumn{3}{|c|}{ CLO } & \multicolumn{2}{|c|}{150} & \multicolumn{3}{|c|}{ KET } & \multirow[t]{2}{*}{$\begin{array}{l}n^{\circ} \\
(*)\end{array}$} & \multicolumn{3}{|c|}{$\mathrm{FLU}_{\text {afm }}$} & \multicolumn{2}{|c|}{$\mathrm{FLU}_{\text {mha }}$} \\
\hline & & & $\mathrm{R}$ & s & \begin{tabular}{l|l}
$\mathrm{I}$ & $\mathrm{R}$ \\
$\mathrm{n}$
\end{tabular} & $\mathrm{s}$ & 1 & $R$ & $s$ & & $\mathrm{R}$ & $s$ & | & $R$ & $s$ & & $\mathbf{R}$ & $s$ & & $\mathrm{R}$ & $\mathrm{s}$ & I & $\mathrm{R}$ & $s$ & & \begin{tabular}{l|l}
$R$ & $S$ \\
$R$
\end{tabular} & & $R$ & & S & \begin{tabular}{|l|l} 
& $R$ \\
\end{tabular} & s & 1 & \\
\hline C. albicans (46) & 46 & - & - & 46 & - & 46 & - & - & 43 & - & 3 & 44 & & 21 & 133 & 31 & 2 & 361 & 10 & - & 25 & 18 & 3 & 44 & 2. & -2 & 320 & 3 & 11 & 1 & -10 & 09 & . & 2 \\
\hline C. glabrata (25) & 25 & - & - & 25 & - & 25 & - & - & 25 & - & - & 25 & - & -2 & 25 & - & - & 24 & 1 & - & 21 & 3 & 1 & 25 & - - & -25 & 5 - & - & 12 & - & -12 & 26 & - & \\
\hline C. parapsilosis (8) & 8 & - & - & 8 & - & 8 & - & - & 6 & - & 2 & 7 & - & 1 & 7 & I & - & 7 & 1 & - & 7 & - & 1 & 8 & - & -8 & - & - & 3 & 2 & -1 & 3 & - & \\
\hline C. krusei (5) & 5 & - & - & 5 & - & 5 & - & - & 3 & 2 & - & 5 & - & - & 5 & - & - & 5 & - & - & 5 & - & - & 5 & - & -4 & 1 & - & 2 & . & -2 & $2-$ & - & \\
\hline C. famata (2) & 2 & - & - & 2 & - & 2 & - & - & 2 & - & - & 2 & - & - & 2 & - & - & 2 & 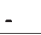 & - & 2 & - & - & 2 & - & -2 & - & - & 1 & - & -1 & - & - & \\
\hline C. guilliermondii (2) & 2 & - & - & 2 & - & 2 & - & - & 1 & 1 & - & 2 & - & - & 2 & - & - & 2 & - & - & 1 & I & - & 2 &.- & -2 & - & - & 1 & - & -1 & - & - & 1 \\
\hline C. tropicalis (2) & 2 & - & - & 2 & - & 2 & - & - & 2 & - & - & 2 & - & - & 1 & I & - & 2 & - & - & 1 & 1 & - & 2 & - & $\begin{array}{l}-2 \\
-2\end{array}$ & - & - & 1 & - & -1 & I- & - & \\
\hline C. humicola (I) & 1 & - & - & 1 & - & 1 & - & - & I & - & - & 1 & - & - & - & 1 & - & 1 & - & - & - & - & 1 & 1 &.- & -1 & - & - & 1 & - & -1 & - & - & 1 \\
\hline C. intermedia (I) & I & - & - & 1 & - & 1 & - & - & I & - & - & 1 & - & - & 1 & - & - & 1 & - & - & 1 & - & - & 1 & - - & -1 & - & - & 1 & - & -1 & 11 & - & - \\
\hline C. kefyr (I) & I & - & - & 1 & - & 1 & - & - & I & - & - & 1 & - & - & 1 & - & - & $\mathrm{I}$ & - & - & I & - & & $\mathrm{I}$ & - & -1 & & - & 1 & - & -1 & 11 & - & - \\
\hline C. lipolytica (I) & I & - & - & 1 & - & 1 & - & - & I & - & - & 1 & - & - & 1 & - & - & 1 & - & - & I & - & - & 1 & - & -1 & 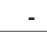 & - & 1 & - & -1 & $1-$ & - & $\mathrm{I}$ \\
\hline C. pulcherrima (I) & I & - & - & 1 & - & I & - & - & I & - & - & 1 & - & - & 1 & - & - & I & - & - & I & - & - & $\mathrm{I}$ & - & -1 & - & - & - & - & $-\quad-$ & - & - & - \\
\hline C. valida (I) & I & - & - & 1 & - & 1 & - & - & I & - & - & 1 & - & - & - & I & - & I & - & - & 1 & - & 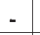 & I &.- & -1 & - & - & - & - & - - & - & - & - \\
\hline C. neoformans (2) & 2 & - & - & 2 & - & 2 & - & - & 2 & - & - & 2 & - & - & 2 & - & - & 2 & - & - & 2 & - & - & 2 & $=$ & -2 & - & - & 2 & 2 & $-\quad-$ & 2 & - & - \\
\hline S. cerevisiae $(10)$ & 10 & - & & 10 & - & 10 & - & 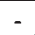 & 10 & & - & 10 & - & - & 9 & I & - & 9 & 1 & - & 6 & I & 3 & 10 & - - & -10 & 0 & - & 5 & 1 & 4 & 45 & - & \\
\hline Pichia carsonii (1) & 1 & - & - & 1 & - & 1 & - & - & 1 & - & - & 1 & - & - & 1 & - & - & $\mathrm{I}$ & - & - & 1 & - & - & $\mathrm{I}$ & - & -1 & - & - & 1 & 1 & - - & -1 & - & - \\
\hline T. cutaneum (4) & 4 & - & - & 4 & - - & 4 & - & - & - & $\mathrm{I}$ & 3 & I & - & 3 & 3 & I & - & 4 & - & - & 4 & - & - & 4 &.- & \begin{tabular}{|l|l}
-4 \\
\end{tabular} & - & - & 4 & 2 & -2 & 23 & 1 & - \\
\hline Rhodotorula spp. (I) & 1 & - & & $\mathrm{I}$ & & 1 & - & - & 1 & & - & 1 & & - & 1 & & - & 1 & - & - & 1 & & & 1 & - & \begin{tabular}{l|l}
-1 & 1
\end{tabular} & - & - & - & - & $-\quad-$ & - & - & \\
\hline
\end{tabular}


Tabella 4. Profili di sensibilità e resistenza agli antimicotici: Ospedale Mauriziano

\begin{tabular}{|c|c|c|c|c|c|c|c|c|c|c|c|c|c|c|c|}
\hline Codice interno & Specie & $\mathrm{AMP}_{20}$ & $\mathrm{AMP}_{100}$ & NYS & $5 \mathrm{FC}_{1}$ & $5 \mathrm{FC}_{10}$ & GRI & $\mathrm{ClC}$ & MIC & ECO & $\mathrm{CLO}$ & 150 & KET & $\mathrm{FLU}_{1000 \mathrm{an}}$ & $\mathrm{FLU}_{100 \text { una }}$ \\
\hline OM 75 & C. albicans & $s$ & s & $s$ & $s$ & $s$ & $\mathrm{R}$ & $s$ & $s$ & $s$ & $s$ & $S$ & $s$ & 1 & 1 \\
\hline OM 89 & C. albicans & $\mathrm{s}$ & $\mathrm{s}$ & $s$ & $\mathrm{~s}$ & $\mathrm{~s}$ & $\mathrm{R}$ & $s$ & 1 & $\mathrm{~s}$ & 1 & $\mathrm{~s}$ & 1 & 1 & 1 \\
\hline OM 81 & C. albicans & $\mathrm{s}$ & 5 & $\mathrm{~s}$ & $\mathrm{~s}$ & $s$ & $\mathrm{R}$ & $\mathrm{s}$ & 1 & 1 & 1 & I & 1 & 1 & 1 \\
\hline OM 80 & C. albicans & $s$ & 5 & $s$ & s & $s$ & $\mathrm{R}$ & $s$ & 1 & 1 & $s$ & $\mathrm{~s}$ & 1 & 1 & 1 \\
\hline OM 83 & C. albicans & $s$ & $\mathrm{~s}$ & $s$ & $s$ & $s$ & $\mathrm{R}$ & $s$ & 1 & s & $s$ & 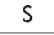 & 1 & 1 & 1 \\
\hline OM 84 & C. albicans & $\mathrm{s}$ & 5 & $\mathrm{~s}$ & $\mathrm{~s}$ & $\mathrm{~s}$ & $\mathrm{R}$ & $\mathrm{s}$ & $\mathrm{s}$ & $\mathrm{s}$ & $s$ & $\mathrm{~s}$ & $\mathrm{~s}$ & 1 & 1 \\
\hline OM 82 & C. albicans & $s$ & $s$ & $s$ & $\mathrm{~s}$ & $\mathrm{~s}$ & $\mathrm{R}$ & $s$ & 1 & $\mathrm{~s}$ & $s$ & $\mathrm{~s}$ & $\mathrm{~s}$ & 1 & 1 \\
\hline OM91 & C. albicans & $\mathrm{s}$ & $\mathrm{s}$ & $s$ & $s$ & $s$ & $\mathrm{R}$ & $s$ & 1 & $s$ & $s$ & $s$ & 1 & 1 & 1 \\
\hline OM 92 & C. albicans & $\mathrm{s}$ & $\mathrm{s}$ & $\mathrm{s}$ & $\mathrm{s}$ & $s$ & $\mathrm{R}$ & $\mathrm{s}$ & 1 & 1 & 1 & S & 1 & 1 & 1 \\
\hline OM 94 & C. albicans & $\mathrm{s}$ & $\mathrm{s}$ & $\mathrm{s}$ & $\mathrm{s}$ & $\mathrm{s}$ & $R$ & $\mathrm{~s}$ & 1 & $\mathrm{~s}$ & $\mathrm{~s}$ & $\mathrm{~s}$ & s & $\mathrm{R}$ & $\mathrm{s}$ \\
\hline OM 95 & C. albicans & $s$ & $s$ & $s$ & $s$ & $s$ & $\mathrm{R}$ & $s$ & 1 & s & $s$ & $s$ & $s$ & $s$ & $s$ \\
\hline OM 98 & C. albicans & $s$ & $s$ & $s$ & $\mathrm{~s}$ & $s$ & $\mathrm{R}$ & $s$ & $\mathrm{R}$ & $s$ & 1 & $s$ & $s$ & $\mathrm{R}$ & $s$ \\
\hline OM 99 & C. albicans & $s$ & $s$ & $s$ & $\mathrm{~s}$ & $s$ & $\mathrm{R}$ & $s$ & $\mathrm{~s}$ & $\mathrm{~s}$ & $s$ & $\mathrm{~s}$ & $s$ & $\mathbf{R}$ & $\mathrm{R}$ \\
\hline OM 108 & C. kefyr & $\mathrm{s}$ & $\mathrm{s}$ & $s$ & $\mathrm{~s}$ & 5 & $\mathrm{R}$ & $\mathrm{s}$ & $s$ & $s$ & s & $\mathrm{s}$ & $\mathrm{s}$ & $\mathrm{R}$ & $\mathrm{s}$ \\
\hline OM 85 & C. valida & $\mathrm{s}$ & $\mathrm{s}$ & $s$ & $\mathrm{~s}$ & $\mathrm{~s}$ & $\mathrm{R}$ & $s$ & 1 & $s$ & $\mathrm{~s}$ & 5 & $\mathrm{~s}$ & 1 & 1 \\
\hline OM 47 & C. glabrata & $\mathrm{s}$ & $\mathrm{s}$ & $\mathrm{s}$ & $\mathrm{s}$ & $\mathrm{s}$ & $\mathrm{R}$ & $s$ & $\mathrm{~s}$ & $s$ & $\mathrm{I}$ & $\mathrm{s}$ & $\mathrm{s}$ & $\mathrm{R}$ & $\mathrm{s}$ \\
\hline OM 49 & C. glabrata & $S$ & 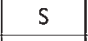 & $S$ & $s$ & $S$ & $\mathrm{R}$ & $s$ & $\mathrm{~s}$ & $\mathrm{~s}$ & $\mathrm{~s}$ & $\mathrm{~s}$ & $\mathrm{~s}$ & $\mathbf{R}$ & $\mathrm{s}$ \\
\hline OM 107 & C. glabrata & $\mathrm{s}$ & s & s & s & $\mathrm{s}$ & $\mathrm{R}$ & $\mathrm{s}$ & $\mathrm{s}$ & $\mathrm{s}$ & $\mathrm{R}$ & S & s & R & $R$ \\
\hline OM 106 & C. glabrata & $s$ & 5 & $s$ & $s$ & $s$ & $\mathrm{R}$ & $s$ & $s$ & $s$ & 1 & s & $s$ & $\mathbf{R}$ & $R$ \\
\hline OM 101 & C. glabrata & $s$ & 5 & $s$ & $s$ & $s$ & $\mathrm{R}$ & $s$ & $s$ & $\mathrm{~s}$ & $s$ & $s$ & $\mathrm{~s}$ & $\mathrm{R}$ & $\mathrm{R}$ \\
\hline OM 51 & C. glabrata & $s$ & $\mathrm{~s}$ & $s$ & $s$ & $\mathrm{~s}$ & $\mathrm{R}$ & $\mathrm{s}$ & $\mathrm{s}$ & $\mathrm{s}$ & 1 & $s$ & $\mathrm{~s}$ & $R$ & $s$ \\
\hline OM 76 & C. glabrata & $\mathrm{s}$ & $\mathrm{s}$ & $s$ & $\mathrm{~s}$ & $\mathrm{~s}$ & $\mathrm{R}$ & $\mathrm{s}$ & $\mathrm{s}$ & $\mathrm{s}$ & $\mathrm{s}$ & $\mathrm{s}$ & $\mathrm{s}$ & 1 & 1 \\
\hline OM 73 & S. cerevisiae & $\mathrm{s}$ & 5 & $\mathrm{~s}$ & $\mathrm{~s}$ & 5 & $\mathrm{R}$ & $s$ & $\mathrm{~s}$ & $\mathrm{~s}$ & $\mathrm{~s}$ & 5 & 5 & 1 & 1 \\
\hline OM 74 & S. cerevisiae & $\mathrm{s}$ & $\mathrm{s}$ & $\mathrm{s}$ & $\mathrm{s}$ & $\mathrm{s}$ & $\mathrm{R}$ & $\mathrm{s}$ & $\mathrm{s}$ & $s$ & $\mathrm{~s}$ & $\mathrm{~s}$ & $\mathrm{~s}$ & 1 & 1 \\
\hline OM 104 & P. carsonii & $\mathrm{s}$ & 5 & $s$ & $s$ & $s$ & $\mathrm{R}$ & $s$ & $\mathrm{~s}$ & $s$ & $s$ & $s$ & $s$ & $\mathrm{~s}$ & $s$ \\
\hline
\end{tabular}

Tabella 5. Profili di sensibilità e resistenza agli antimicotici: Ospedale O.I.R.M.- Sant'Anna.

\begin{tabular}{|c|c|c|c|c|c|c|c|c|c|c|c|c|c|c|c|}
\hline Codice interno & Specie & $\mathrm{AMP}_{20}$ & $\mathrm{AMP}_{100}$ & NYS & $5 \mathrm{FC}_{1}$ & $5 \mathrm{FC}_{10}$ & GRI & $\mathrm{ClC}$ & MIC & ECO & CLO & ISO & KET & $F L U_{100 a t m}$ & $\mathrm{FLU}_{100 \mathrm{mha}}$ \\
\hline OIRM 477 & C. albicans & $\mathrm{s}$ & $\mathrm{S}$ & $\mathrm{s}$ & $\mathrm{s}$ & $s$ & $\mathrm{R}$ & $\mathrm{s}$ & $\mathrm{s}$ & $\mathrm{s}$ & 1 & $\mathrm{~s}$ & $\mathrm{~s}$ & $R$ & $\mathrm{~s}$ \\
\hline OIRM 426 & C. albicans & $s$ & $s$ & $s$ & $R$ & $\mathrm{~s}$ & $R$ & $s$ & $\mathrm{~s}$ & $s$ & 1 & $s$ & 1 & 1 & 1 \\
\hline OIRM 433 & C. albicans & $\mathrm{s}$ & $s$ & $s$ & $s$ & $s$ & $R$ & $s$ & 1 & $s$ & 1 & $s$ & 1 & 1 & 1 \\
\hline OIRM 456 & C. albicans & $s$ & $\mathrm{~s}$ & s & $s$ & $s$ & $\mathrm{R}$ & $s$ & 1 & $s$ & 1 & $s$ & s & 1 & 1 \\
\hline OIRM 427 & C. albicans & $s$ & $\mathrm{~s}$ & 5 & $s$ & $s$ & $R$ & $s$ & 1 & $S$ & $s$ & $s$ & $s$ & 1 & 1 \\
\hline OIRM 421 & C. albicans & s & $s$ & $\mathrm{~s}$ & $\mathrm{~s}$ & $s$ & $\mathrm{R}$ & $\mathrm{s}$ & 1 & $\mathrm{~s}$ & 1 & $S$ & $R$ & $\mathrm{R}$ & $s$ \\
\hline OIRM 420 & C. albicans & $s$ & $\mathrm{~s}$ & 5 & $s$ & $s$ & $\mathbf{R}$ & $s$ & 1 & $s$ & $\mathrm{~s}$ & $S$ & 1 & 1 & 1 \\
\hline OIRM 44 & C. albicans & $S$ & $S$ & $s$ & $S$ & $s$ & $R$ & $s$ & $s$ & $S$ & $\mathrm{~s}$ & $s$ & $\mathrm{~s}$ & 1 & 1 \\
\hline OIRM 437 & C. krusei & s & $S$ & $s$ & $S$ & $s$ & $\mathbf{R}$ & $s$ & $S$ & $S$ & $s$ & $S$ & $S$ & $R$ & $R$ \\
\hline OIRM 468 & C. krusei & $\mathrm{s}$ & $\mathrm{s}$ & $\mathrm{s}$ & 1 & $s$ & $\mathrm{R}$ & $\mathrm{s}$ & $\mathrm{s}$ & $\mathrm{s}$ & $\mathrm{s}$ & $\mathrm{s}$ & 1 & $R$ & $\mathrm{R}$ \\
\hline OIRM 422 & C. krusei & $\mathrm{s}$ & $s$ & $\mathrm{~s}$ & $s$ & $s$ & $R$ & $s$ & $s$ & $s$ & s & $s$ & $\mathrm{~s}$ & 1 & 1 \\
\hline OIRM 464 & C. parapsilosis & $s$ & $\mathrm{~s}$ & s & $s$ & $s$ & $R$ & $\mathrm{~s}$ & $\mathrm{~s}$ & $s$ & s & $S$ & $\mathrm{~s}$ & $s$ & $s$ \\
\hline OIRM 443 & C. tropicalis & $s$ & $s$ & $s$ & $s$ & $s$ & $R$ & 5 & $s$ & $\mathrm{~s}$ & $s$ & $s$ & $s$ & 1 & 1 \\
\hline OIRM 440 & C. glabrata & $s$ & $\mathrm{~s}$ & $s$ & $s$ & $S$ & $R$ & $s$ & $S$ & $s$ & $s$ & $S$ & $\mathrm{~s}$ & 1 & 1 \\
\hline OIRM 476 & C. glabrata & $s$ & $s$ & $s$ & $s$ & $s$ & $R$ & $S$ & $s$ & $s$ & 5 & $s$ & $s$ & $R$ & $s$ \\
\hline OIRM 484 & C. glabrata & $\mathrm{s}$ & $S$ & 5 & $\mathrm{~s}$ & $S$ & $\mathbf{R}$ & $S$ & $S$ & $S$ & $s$ & $S$ & $\mathrm{~s}$ & $R$ & 5 \\
\hline OIRM 417 & C. glabrata & $S$ & $\mathrm{~s}$ & $\mathrm{~s}$ & $\mathrm{~s}$ & $s$ & $R$ & 5 & 5 & $\mathrm{~s}$ & $s$ & 5 & $s$ & 1 & 1 \\
\hline OIRM 487 & S. cerevisiae & $s$ & 5 & 5 & 5 & $s$ & $R$ & $s$ & $s$ & $s$ & 1 & 5 & $s$ & $\mathrm{R}$ & 5 \\
\hline OIRM 494 & S. cerevisiae & $s$ & $s$ & $\mathrm{~s}$ & $s$ & $s$ & $\mathrm{R}$ & 5 & I & 1 & $\mathrm{R}$ & $S$ & $s$ & $\mathrm{R}$ & $s$ \\
\hline OIRM 45I & S. cerevisiae & $\mathrm{s}$ & $\mathrm{s}$ & $s$ & $\mathrm{~s}$ & $s$ & $\mathrm{R}$ & $s$ & $\mathrm{~s}$ & $\mathrm{~s}$ & $\mathrm{~s}$ & $\mathrm{~s}$ & $\mathrm{~s}$ & $s$ & $s$ \\
\hline OIRM 460 & S. cerevisiae & $\mathrm{s}$ & $S$ & $\mathrm{~s}$ & $\mathrm{~s}$ & $\mathrm{~s}$ & $R$ & $S$ & $\mathrm{~s}$ & $S$ & $R$ & $S$ & $\mathrm{~s}$ & $R$ & 5 \\
\hline OIRM 439 & S. cerevisiae & $s$ & $s$ & $s$ & $s$ & $s$ & $\mathrm{R}$ & 5 & $s$ & $s$ & $s$ & $s$ & $s$ & 1 & 1 \\
\hline ORM 465 & S. cerevisiae & $s$ & $\mathrm{~s}$ & $s$ & $s$ & $s$ & $\mathbf{R}$ & $s$ & $S$ & $s$ & $\mathrm{~s}$ & $S$ & $S$ & $\mathrm{R}$ & $s$ \\
\hline
\end{tabular}


Tabella 6. Profili di sensibilità e resistenza agli antimicotici: Ospedale San Lazzaro

\begin{tabular}{|c|c|c|c|c|c|c|c|c|c|c|c|c|c|c|c|}
\hline Codice interno & Specie & AMP $_{20}$ & $\mathrm{AMP}_{100}$ & NYS & $5 \mathrm{FC}_{1}$ & $5 \mathrm{FC}_{10}$ & GRI & $\mathrm{ClC}$ & MIC & ECO & CLO & 150 & KET & $\mathrm{FLU}_{10 \mathrm{catin}}$ & $\mathrm{FLU}_{100 \mathrm{~m} \text { ha }}$ \\
\hline SL 237 & C. albicans & $\mathrm{s}$ & $\mathrm{s}$ & $s$ & $s$ & $s$ & $R$ & $S$ & 1 & 1 & $R$ & $\mathrm{~s}$ & $\mathrm{~s}$ & 1 & 1 \\
\hline SL 231 & C. albicans & $s$ & $s$ & $s$ & $s$ & $s$ & $\mathrm{R}$ & $s$ & 1 & $s$ & $s$ & $s$ & 1 & 1 & 1 \\
\hline SL 235 & C. albicans & $\mathrm{s}$ & $S$ & $\mathrm{~s}$ & $\mathrm{~s}$ & 5 & $\mathrm{R}$ & $s$ & 1 & 1 & 1 & $S$ & 1 & 1 & 1 \\
\hline SL $632 / 02$ & C. albicans & $\mathrm{s}$ & $\mathrm{s}$ & $s$ & s & s & $R$ & $\mathrm{~s}$ & $R$ & $\mathrm{~s}$ & 1 & $S$ & I & $R$ & $\mathbf{R}$ \\
\hline SL $640 / 02$ & C. famata & $\mathrm{s}$ & $\mathrm{s}$ & $s$ & $s$ & $s$ & $R$ & $s$ & $s$ & $\mathrm{~S}$ & $\mathrm{~s}$ & $s$ & $s$ & $\mathrm{R}$ & $R$ \\
\hline SL 230 & C. famata & $s$ & $s$ & $s$ & $s$ & $s$ & $R$ & $s$ & $s$ & $S$ & $S$ & $S$ & $s$ & 1 & 1 \\
\hline SL $611 / 02$ & C. guilliermondii & $\mathrm{s}$ & $\mathrm{S}$ & $s$ & 5 & 5 & $\mathrm{R}$ & $s$ & $s$ & $\mathrm{~s}$ & $\mathrm{~s}$ & $S$ & $s$ & $\mathrm{R}$ & $\mathrm{R}$ \\
\hline SL $614 / 02$ & C. humicola & $\mathrm{s}$ & $S$ & $\mathrm{~s}$ & $s$ & $s$ & $R$ & $s$ & 1 & $S$ & $R$ & $s$ & $s$ & $\mathrm{R}$ & $R$ \\
\hline SL 639/02 & C. intermedia & $\mathrm{s}$ & $S$ & $s$ & 5 & $s$ & $R$ & $\mathrm{~s}$ & $s$ & $s$ & $S$ & $\mathrm{~s}$ & $\mathrm{~s}$ & $R$ & $\mathrm{~s}$ \\
\hline SL $63 \mathrm{I} / 02$ & C. lipolytica & $\mathrm{s}$ & $\mathrm{s}$ & $\mathrm{s}$ & 5 & $s$ & 5 & $s$ & $s$ & $\mathrm{~s}$ & $\mathrm{~s}$ & $S$ & $s$ & $\mathrm{R}$ & $\mathrm{R}$ \\
\hline SL 636/02 & C. parapsilosis & $\mathrm{s}$ & $\mathrm{S}$ & $\mathrm{s}$ & $s$ & $s$ & $R$ & $\mathrm{~S}$ & $\mathrm{~s}$ & $\mathrm{~s}$ & $\mathrm{~s}$ & $\mathrm{~s}$ & $\mathrm{~s}$ & $R$ & $\mathrm{~S}$ \\
\hline SL 24I & C. parapsilosis & $\mathrm{s}$ & $s$ & $S$ & $s$ & $s$ & $R$ & $s$ & $s$ & $s$ & $\mathrm{~s}$ & 5 & $s$ & 1 & 1 \\
\hline SL 236 & C. parapsilosis & $s$ & $s$ & $s$ & 5 & $s$ & $R$ & $S$ & $s$ & 1 & $s$ & $s$ & $s$ & 1 & 1 \\
\hline SL 232 & C. parapsilosis & $\mathrm{s}$ & $S$ & $\mathrm{~s}$ & 5 & $s$ & $R$ & $s$ & $s$ & $s$ & $s$ & $S$ & $\mathrm{~s}$ & 1 & 1 \\
\hline SL $613 / 02$ & C. tropicalis & $s$ & $\mathrm{~s}$ & $\mathrm{~s}$ & $\mathrm{~s}$ & $s$ & $\mathrm{R}$ & 5 & 1 & $S$ & 1 & $s$ & $s$ & $\mathrm{R}$ & $R$ \\
\hline SL 242 & S. cerevisiae & $\mathrm{s}$ & $S$ & $\mathrm{~S}$ & $\mathrm{~s}$ & $s$ & $R$ & $\mathrm{~s}$ & $\mathrm{~s}$ & $\mathrm{~s}$ & $R$ & $\mathrm{~S}$ & $\mathrm{~s}$ & 1 & 1 \\
\hline SL 233 & Rhodotorula spp. & $\mathrm{s}$ & $\mathrm{s}$ & $\mathrm{s}$ & 5 & 5 & $\mathrm{R}$ & $s$ & $\mathrm{~s}$ & $\mathrm{~s}$ & $\mathrm{~s}$ & $\mathrm{~s}$ & $S$ & 1 & 1 \\
\hline SL 628/02 & T. cutaneum & $\mathrm{s}$ & $s$ & $\mathrm{~s}$ & 1 & $s$ & $R$ & $S$ & $\mathrm{~s}$ & $\mathrm{~s}$ & $\mathrm{~s}$ & $s$ & $s$ & $s$ & $s$ \\
\hline SL $17 / 02$ & T. cutaneum & $\mathrm{s}$ & $S$ & $\mathrm{~s}$ & $R$ & $\mathrm{R}$ & $R$ & $s$ & 1 & $s$ & $s$ & $S$ & $\mathrm{~s}$ & $\mathrm{R}$ & $\mathrm{s}$ \\
\hline SL 524/02 & T. cutaneum & $s$ & $\mathrm{~s}$ & $S$ & $R$ & $R$ & $R$ & $S$ & $\mathrm{~s}$ & $s$ & $S$ & $s$ & $s$ & $R$ & $R$ \\
\hline SL 507/02 & T. cutaneum & $\mathrm{s}$ & $\mathrm{s}$ & $\mathrm{s}$ & $\mathrm{R}$ & $R$ & $\mathrm{R}$ & $\mathrm{s}$ & $\mathrm{s}$ & $s$ & 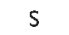 & $s$ & $s$ & $\mathrm{~s}$ & $s$ \\
\hline
\end{tabular}

Tabella 7. Profili di sensibilità e resistenza agli antimicotici: Ospedale Molinette

\begin{tabular}{|c|c|c|c|c|c|c|c|c|c|c|c|c|c|c|c|}
\hline Codice interno & Specie & $\mathrm{AMP}_{20}$ & $\mathrm{AMP}_{100}$ & NYS & $5 \mathrm{FC}_{1}$ & $5 \mathrm{FC}_{10}$ & GRI & $\mathrm{ClC}$ & $\mathrm{MIC}$ & $\mathrm{ECO}$ & $\mathrm{CLO}$ & ISO & KET & $\mathrm{FLU}_{100 \mathrm{~atm}}$ & $\mathrm{FLU}_{100 \mathrm{mha}}$ \\
\hline MOL 52 & C. albicans & $S$ & $S$ & $s$ & $s$ & $\mathrm{~s}$ & $R$ & $S$ & 1 & $S$ & 1 & $s$ & $s$ & 1 & 1 \\
\hline MOL 50 & C. albicans & $\mathrm{s}$ & 5 & $s$ & $\mathrm{~s}$ & $\mathrm{~s}$ & $R$ & $s$ & $\mathrm{~s}$ & 5 & $\mathrm{~s}$ & $s$ & $\mathrm{~s}$ & 1 & 1 \\
\hline MOL 55 & C. albicans & $\mathrm{S}$ & 5 & $S$ & $\mathrm{~s}$ & $\mathrm{~S}$ & $R$ & $s$ & 1 & 1 & I & 1 & 1 & 1 & 1 \\
\hline MOL 59 & Calbicans & $\mathrm{s}$ & $\mathrm{s}$ & $s$ & $\mathrm{~s}$ & $\mathrm{~s}$ & $\mathrm{R}$ & $s$ & 5 & $\mathrm{~s}$ & $s$ & $s$ & $\mathbf{I}$ & 1 & 1 \\
\hline MOL 56 & C. albicans & $\mathrm{s}$ & $\mathrm{s}$ & $s$ & $\mathrm{~s}$ & $\mathrm{~s}$ & $R$ & $s$ & s & $\mathrm{s}$ & $s$ & $\mathrm{~s}$ & $\mathrm{~s}$ & 1 & 1 \\
\hline MOL 6I & C.albicans & $\mathrm{s}$ & $\mathrm{s}$ & $\mathrm{s}$ & $\mathrm{s}$ & $\mathrm{s}$ & $R$ & $\mathrm{~s}$ & $\mathrm{~s}$ & $\mathrm{~s}$ & $\mathrm{~s}$ & $\mathrm{~s}$ & $\mathrm{~s}$ & 1 & 1 \\
\hline MOL 62 & C. albicans & $\mathrm{s}$ & 5 & $s$ & $\mathrm{~s}$ & 5 & $R$ & $s$ & $\mathrm{~s}$ & $\mathrm{~s}$ & s & $\mathrm{s}$ & $\mathrm{s}$ & 1 & 1 \\
\hline MOL 57 & C. albicans & $\mathrm{s}$ & $\mathrm{s}$ & $s$ & $\mathrm{~s}$ & $\mathrm{~s}$ & $R$ & $s$ & $\mathrm{~s}$ & I & $\mathrm{s}$ & $\mathrm{s}$ & $\mathrm{s}$ & 1 & 1 \\
\hline MOL 66 & C. albicans & $s$ & $s$ & $s$ & s & $\mathrm{s}$ & $R$ & $s$ & 1 & $\mathrm{~s}$ & s & $s$ & $\mathrm{~s}$ & $R$ & $\mathrm{~s}$ \\
\hline MOL 58 & C. glabrata & $s$ & $\mathrm{~s}$ & $s$ & s & $\mathrm{s}$ & $R$ & $s$ & s & $\mathrm{s}$ & $s$ & $s$ & $\mathrm{~s}$ & 1 & 1 \\
\hline MOL 54 & C. glabrata & $\mathrm{s}$ & 5 & $\mathrm{~s}$ & 5 & 5 & $R$ & $s$ & 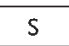 & $s$ & s & $\mathrm{s}$ & $\mathrm{s}$ & 1 & 1 \\
\hline MOL 3 & C. glabrata & s & $s$ & $s$ & $s$ & s & $R$ & $s$ & $s$ & I & s & $s$ & s & 1 & 1 \\
\hline MOL 6 & C. glabrata & $\mathrm{s}$ & $s$ & $s$ & $\mathrm{~s}$ & $\mathrm{~s}$ & $R$ & $s$ & $s$ & s & $s$ & $s$ & s & 1 & 1 \\
\hline MOL 7 & C. glabrata & $s$ & 5 & $s$ & $s$ & 5 & $R$ & $s$ & $s$ & $s$ & $s$ & $s$ & s & 1 & 1 \\
\hline MOL 15 & C. glabrata & $s$ & $s$ & $s$ & $\mathrm{~s}$ & $s$ & $R$ & $s$ & $S$ & $s$ & $s$ & $s$ & $s$ & 1 & 1 \\
\hline MOL 65 & C. glabrata & $s$ & 5 & $s$ & $s$ & 5 & $R$ & $s$ & $s$ & $s$ & $s$ & $s$ & $s$ & $R$ & $R$ \\
\hline MOL 53 & C. glabrata & $s$ & $s$ & $s$ & $s$ & $s$ & $R$ & $s$ & $s$ & $s$ & $s$ & $s$ & $s$ & 1 & 1 \\
\hline MOL 19B & C. glabrata & $s$ & $\mathrm{~s}$ & $s$ & $s$ & $s$ & $R$ & $s$ & 5 & $s$ & $s$ & $s$ & $s$ & $R$ & $R$ \\
\hline MOL 60 & C. glabrata & $S$ & s & $s$ & $s$ & $\mathrm{~s}$ & $R$ & $s$ & $s$ & s & $s$ & $s$ & $s$ & 1 & 1 \\
\hline
\end{tabular}




\section{BIBLIOGRAFIA}

1. Alvarado D, Diaz MC, Silva V. Identification and antifungal susceptibility of Candida spp. isolated from invasive mycoses. Influence of growth inhibition percentage to determine minimal inhibitory concentration. Rev Med Chil 2002; 130: 416-23.

2. Anaissie E. Opportunistic mycoses in the immunocompromised host: experience at a cancer center and review. Clin Inf Dis 1992; 14 (suppl. 1): S43-S53.

3. Arikan S. Lipid-based antifungal agents: a concise overview. Cell Mol Biol Lett 2002; 7: 919-22.

4. Blot SI, Vandewoude KH, Hoste EA, Colardyn FA. Effects of nosocomial candidemia on outcomes of critically III patients. Am J Med 2002; 113: 480-5.

5. Boschman CR, Bodnar UR, Tornatore MA et al. Thirteen-year evolution of azole resistance in yeast isolates and prevalence of resistant strains carried by cancer patients at a large medical center. Antimicrob Agents Chemother 1998; 42: 734-38.

6. Cinar S, Nedret Koc A, Taskapan H, Dogukan A, Tokgoz B, Utas C. Case report. Candida lusitaniae peritonitis in a patient on continuous ambulatory peritoneal dialysis. Mycoses 2002; 45: 120-2.

7. Colombo AL, Da Matta D, De Almeida LP, Rosas R. Fluconazole susceptibility of brazilian Candida isolates assessed by a disk diffusion method. Braz J Infect Dis 2002; 6: 118-23.

8. Espinel-Ingroff A, Boyle K, Sheehan DJ. In vitro antifungal activities of voriconazole and reference agents as determined by NCCLS methods: review of the literature. Mycopathologia 2001; 150: 101-15.

9. Faggi E, Farina C, Manso E, et al. Funguria nei pazienti ospedalizzati: indagine retrospettiva multicentrica. Microbiol Med 2004; 19: 389-92.

10. Garcia-Martos P, Dominguez I, Marin P, GarciaAgudo R, Aoufi S, Mira J. Antifungal susceptibility of emerging yeast pathogens. Enferm Infect Microbiol Clin 2001; 19: 249-56.

11. Ghannoum MA, Rice LB. Antifungal agents: mode of action, mechanisms of resistance, and correlation of these mechanisms with bacterial resistance. Clin Microbiol Rev 1999; 12: 501-17.

12. Gudlaugsson O, Gillespie S, Lee K, et al. Attributable mortality of nosocomial candidemia, revisited. Clin Infect Dis 2003; 37: 1172-7.

13. Hossain MA, Ghannoum MA. New investigational antifungal agents for treating invasive fungal infections. Expert Opin Investig Drugs 2000; 9: 1797-813.

14. Krcmery V, Barnes AJ. Non-albicans Candida spp. causing fungaemia: pathogenicity and antifungal resistance. J Hosp Infect 2002; 50: 243-60.

15. Liebowitz LD, and Global Antifungal Surveillance Group (Carlone NA, Tullio V). A two year global evalutation of the susceptibility of Candida species to fluconazole by disk diffusion. Diagn Microbiol Inf Dis 2001; 4: 27-33.

16. Majoros L, Kardos G, Pocsi I, Szabo B. Distribution and susceptibility of Candida spp. isolated in the Medical University of Debrecen. Acta Microbiol Immunol Hung 2002; 49: 351-61.

17. Meis J, Petrou M, Bille J, Ellis D, Gibbs D, And The Global Antifungal Surveillance Group. A global evaluation of the susceptibility of Candida species to fluconazole by disk diffusion. Diagn Microbiol Infect Dis 2000; 36: 215-23.

18. Meunier F, Aoun M, Bitar N. Candidemia in immunocompromised patients. Clin Inf Dis 1992; 14 (suppl. 1): S120-S6.
19. National Committee For Clinical Laboratory Standard. Reference method for broth dilution antifungal susceptibility testing of yeasts; approved standard. M27-A. NCCLS 1996, Villanova, PA.

20. National Committee For Clinical Laboratory Standard. Method for antifungal disk diffusion susceptibility testing of yeasts; proposed guidelines. M44-P, NCCLS 2003, Villanova, PA.

21. Neely MN, Ghannoum MA. The exciting future of antifungal therapy. Eur J Clin Microbiol Infect Dis 2000; 19: 897-914.

22. Pfaller M, Wenzel R. Impact of the changing epidemiology of fungal infections in the 1990s Eur J Clin Microbiol Infect Dis 1992; 11: 287-91.

23. Rex JH, Pfaller MA. Has antifungal susceptibility testing come age? Clin Infect Dis 2002; 35: 982-89.

24. Rogers TR. Optimal use of existing and new antifungal drugs. Curr Opin Crit Care 2001; 7: 238-41.

25. St Georgiev V. Membrane transporters and antifungal drug resistance. Curr Drug Targets 2000; 1: 261-84.

26. Tumbarello M, Tacconelli E, De Gaetano Donati K, Morace G, Fadda G, Cauda R. Candidemia in HIVinfected subjects. Eur J Clin Microbiol Infect Dis 1999; 18: 478-83.

27. Walsh TJ, Groll AH. Emerging fungal pathogens: evolving challenges to immunocompromised patients for the twenty-first century. Transpl Inf Dis 1999; 1 : 247-61.

Vivian Tullio

Dipartimento di Sanità Pubblica e Microbiologia

Via Santena 9 - 10126 Torino

Tel. 011.6705637 - Fax 011.2365637

E-mail: vivian.tullio@unito.it 